\title{
Visualização 3D interativa de dados médicos temporais baseada em modelo de atenção visual
}

\author{
Leonardo S. Silva \\ Interlab - EPUSP e LApIS - EACH/USP \\ Universidade de São Paulo - USP \\ São Paulo, Brasil \\ leonardosilva@usp.br
}

\author{
Ricardo Nakamura \\ Interlab/EPUSP \\ Universidade de São Paulo - USP \\ São Paulo, Brasil \\ ricardonakamura@usp.br
}

\author{
Fatima L. S. Nunes \\ LApIS - EACH/USP e Interlab - EPUSP \\ Universidade de São Paulo - USP \\ São Paulo, Brasil \\ fatima.nunes@usp.br
}

\begin{abstract}
Resumo-A visualização de dados temporais oferece suporte a processos de planejamento e de decisão ao ajudar na compreensão dos padrões e relacionamento entre tais dados. Na área da Saúde, o procedimento de anamnese produz um grande volume de valiosas informações, que são normalmente analisadas de acordo com aspectos temporais. Abordagens baseadas em técnicas de Realidade Virtual (RV) para visualização de dados temporais ainda podem ser consideradas subexploradas. Neste contexto, também merece destaque o crescimento em importância da Atenção Visual em diversas áreas de conhecimento, em função de sua capacidade de auxiliar seres humanos a explorar complexas cenas visuais. Um projeto apropriado pode favorecer a interpretação de informações complexas em uma visualização, dessa forma, além do uso da Atenção Visual no projeto de uma representação visual para dados médicos com características temporais em um ambiente 3D interativo, busca-se estabelecer uma relação entre os elementos gráficos utilizados e as preferências do usuário por meio de técnicas de aprendizagem e máquina e reconhecimento de padrões. A partir dessa relação, objetiva-se contribuir com a melhoria da percepção visual nas ferramentas de visualização de informação, bem como avançar na personalização das visualizações oferecidas a partir das preferências dos usuários. Resultados preliminares apontam que a Atenção Visual pode ser um recurso importante no projeto de visualização de informações, além de sinalizarem possibilidades de pesquisa na exploração de técnicas de aprendizagem de máquina no contexto do projeto das interfaces dessas visualizações.
\end{abstract}

Index Terms-Visualização de informação, dados temporais, atenção visual, aprendizagem de máquina.

\section{INTRODUÇÃO}

A Visualização de Informação (VI) tornou-se um importante campo de estudo por viabilizar ferramentas que permitam manipular e explorar dados, estabelecendo uma interface entre a mente humana e o poder oferecido pelos avanços tecnológicos. Oferecendo assim, a possibilidade de representação visual para dados com características abstratas e consequentemente sua compreensão e manipulação [1].

$\mathrm{Na}$ área médica, muitos desses dados apresentam um forte aspecto temporal, tendo seus valores alterados ao longo do tempo [2], [3]. O uso de técnicas de Realidade Virtual (RV) na área da saúde vem criando diversas possibilidades de aplicações, além de avanços relacionados ao desenvolvimento de hardware e software [4]. Entretanto, ainda que possa trazer relevantes contribuições a visualização e exploração de dados temporais, seu uso ainda pode ser considerado limitado tendo em vista que grande parte dos métodos utilizados para visualizar dados baseiam-se em técnicas bidimensionais bem conhecidas [5].

Considerando sua habilidade em ajudar seres humanos a processar e explorar cenas visuais complexas, por exemplo, orientando a localização de uma região de interesse, a Atenção Visual tem recebido grande atenção da comunidade científica [6]-[8].

Por essa razão, este trabalho busca contribuir com o aumento da percepção visual das informações em um ambiente tridimensional (3D) interativo para visualização de dados temporais, buscando suporte junto a aplicação do modelo de Atenção Visual no projeto da representação visual e no uso de técnicas de aprendizado de máquina para estabelecer uma relação entre os elementos gráficos e as preferências do usuário.

\section{MotivaÇÃo}

Um levantamento realizado por meio de uma Revisão Sistemática de Literatura apontou que o espaço bidimensional (2D) tem sido majoritariamente escolhido para construção de representações visuais para técnicas de visualização de dados temporais. Técnicas baseadas no uso do espaço 3D ou híbridas (2D e 3D), representam um pequeno número de iniciativas e que compreendem em sua maioria, extensões as técnicas tradionalmente usadas no espaço 2D.

Mecanismos de Atenção Visual contribuem com a redução do esfoço cognitivo envolvido na execução de uma tarefa, ao favorecer a seleção de áreas de interesse, saliências, dentre outros aspectos visuais [9]. A Atenção Visual tem sido amplamente utilizada em conjunto com técnicas de aprendizagem de máquina no auxilio a segmentação de objetos, reconhecimento de objetos, geração de legendas para imagens, dentre outras atvidades [6].

$\mathrm{O}$ uso do aprendizado de máquina não é exatamente uma novidade em VI, sendo empregado na seleção de conteúdo e descoberta de padrões nos dados. Entretanto, seu uso no projeto de interfaces para visualização de informações ainda é pouco explorado. 


\section{TRABALhos CORRELATOS}

Alguns trabalhos buscam auxiliar na compreensão da grande variedade de aplicações existentes [10], apontando a falta de pesquisa relacionando técnicas visuais e dados temporais. Posteriormente, com a introdução da questão do uso de Visual Analytics a discussão sobre técnicas de visualização de dados temporais foi estendida por meio de uma categorização alinhada à identificação de futuras tarefas de Visual Analytics [2].

Em uma ferramenta de visualização de informação, elementos como: projeto, memória, atenção visual e percepção, podem afetar a forma como o usuário observa a visualização. Entretanto, o uso de modelo de atenção visual para predizer os pontos de fixação durante uma análise exploratória, ou mesmo, a influência que estimulos de saliências tem na análise visual para execução de uma tarefa, possuem poucas evidências documentadas, abrindo espaço para interessantes oportunidades de pesquisa [11].

\section{SoluÇÃo Proposta}

A partir do desenvolvimento de um estudo de caso, definiuse que a representação visual a ser utilizada seria inspirada no formato de um poliedro (Figura 1). Inicialmente, a representação visual será configurada com dados oriundos de um banco de dados e agrupados em quatro dimensões: Sintomas, Diagnóstico, Conduta e Evolução.

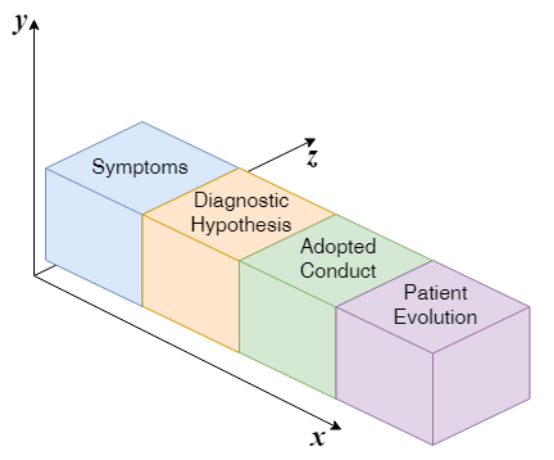

Figura 1. Representação visual escolhida para visualização de dados médicos

O poliedro formado por todas as consultas de um paciente, representa o histórico médico desse paciente. As dimensões que formam os dados de uma consulta individual (Figura 1) são alocadas ao longo do Eixo $X$. Por sua vez, o Eixo $Y$ é usado para registrar o valor, todas as consultas individuais do paciente são alinhadas ao longo do Eixo $Z$, permitindo assim estabelecer uma linha do tempo.

Os elementos gráficos usados na concepção do protótipo foram guiados com base no Modelo de Atenção Visual, de forma que cada dimensão seja identificada de maneira exclusiva por uma cor exclusiva e por uma forma geométrica não-exclusiva. As informações que possuam relevância para o usuário podem ser destacadas de três formas: por tamanho, por cor complementar ou por contraste.

\section{Resultados Preliminares}

A partir de dados de anamnese oriundos do serviço ambulatorial, um protótipo foi implementado e submetido a um Teste de Usabilidade e a um experimento. Dessa forma, cada usuário avaliou 45 diferentes combinações entre os elementos gráficos projetados a partir do Modelo de Atenção Visual, devendo manifestar sua aprovação ou desaprovação.

O conjunto de dados formado pelas respostas foi então usado como entrada para técnicas de aprendizagem baseada em regras, com intenção de estabelecer uma relação entre os elementos gráficos usados e as preferências dos usuários.

\section{CONCLUSÃO}

Os resultados preliminares apontaram a existência de um conjunto de regras que relacionam os elementos gráficos. Essas regras orientarão a geração de novas variações para visualização proposta. Espera-se que essas novas visualizações sejam mais significativas aos usuários, favorecendo assim, a melhor percepção das informações apresentadas.

O conjunto de dados também será usado no treinamento de um classificador, baseado em árvore de decisão, a ser usado futuramente na avaliação das novas variações da visualização. Objetiva-se com isso, estabelecer um mecanismo permanente para aprimoramento da visualização para um determinado grupo de usuários ou, no caso, de um novo domínio de aplicação, que permita ajustar a visualização as preferencias desse novo grupo.

\section{REFERÊNCIAS}

[1] S. LIU, W. CUI, Y. WU, and M. LIU, "A survey on information visualization: Recent advances and challenges," The Visual Computer, 2014.

[2] W. Aigner, S. Miksch, W. Müller, H. Schumann, and C. Tominski, "Visualizing time-oriented data-a systematic view," Computers \& Graphics, vol. 31, no. 3, pp. 401 - 409, 2007. [Online]. Available: http://www.sciencedirect.com/science/article/pii/S0097849307000611

[3] R. Morawa, T. Horak, U. Kister, A. Mitschick, and R. Dachselt, "Combining timeline and graph visualization," in Proceedings of the Ninth ACM International Conference on Interactive Tabletops and Surfaces, ser. ITS '14. New York, NY, USA: ACM, 2014, pp. 345-350. [Online]. Available: http://doi.acm.org/10.1145/2669485.2669544

[4] F. L. S. NUNES, R. M. E. M. COSTA, L. S. MACHADO, and R. M. MORAES, "Realidade virtual para sáude no brasil: Conceitos, desafios e oportunidades," Revista Brasileira de Engenharia Biomédica, vol. 27, no. $4,2011$.

[5] A. Kirk, Data Visualization: a successful design process. Packt Publishing Ltd, 2012.

[6] X. Liu and M. Milanova, "Visual attention in deep learning: a review [j]," Int Rob Auto J, vol. 4, no. 3, pp. 154-155, 2018.

[7] A. M. Treisman and G. Gelade, "A feature-integration theory of attention," Cognitive psychology, vol. 12, no. 1, pp. 97-136, 1980.

[8] A. Borji and L. Itti, "State-of-the-art in visual attention modeling," IEEE transactions on pattern analysis and machine intelligence, vol. 35, no. 1, pp. 185-207, 2013.

[9] S. Frintrop, E. Rome, and H. I. Christensen, "Computational visual attention systems and their cognitive foundations: A survey," $A C M$ Transactions on Applied Perception (TAP), vol. 7, no. 1, p. 6, 2010

[10] S. F. Silva and T. Catarci, "Visualization of linear time-oriented data: a survey," in Proceedings of the First International Conference on Web Information Systems Engineering, vol. 1, 2000, pp. 310-319 vol.1.

[11] P. Polatsek, M. Waldner, I. Viola, P. Kapec, and W. Benesova, "Exploring visual attention and saliency modeling for task-based visual analysis," Computers \& Graphics, vol. 72, pp. 26-38, 2018. 